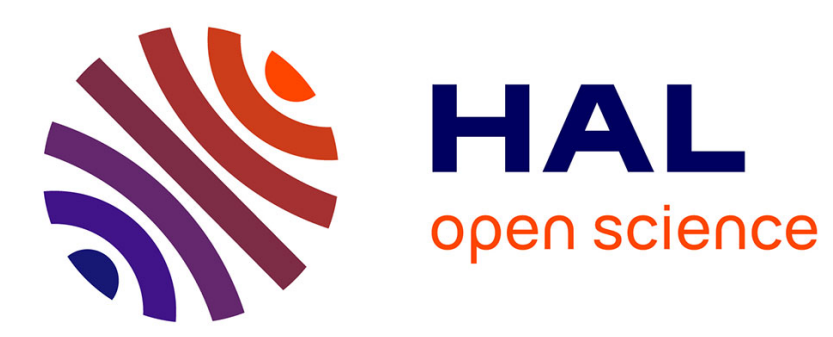

\title{
Le complexe palatial alto-médiéval de Saint-Projet (Bordeaux, Gironde)
}

Frédéric Boutoulle, Ezéchiel Jean-Courret

\section{To cite this version:}

Frédéric Boutoulle, Ezéchiel Jean-Courret. Le complexe palatial alto-médiéval de Saint-Projet (Bordeaux, Gironde). Aquitania, 2016, 32, pp.267-282. hal-01453148

\author{
HAL Id: hal-01453148 \\ https://hal.science/hal-01453148
}

Submitted on 22 Jul 2021

HAL is a multi-disciplinary open access archive for the deposit and dissemination of scientific research documents, whether they are published or not. The documents may come from teaching and research institutions in France or abroad, or from public or private research centers.
L'archive ouverte pluridisciplinaire HAL, est destinée au dépôt et à la diffusion de documents scientifiques de niveau recherche, publiés ou non, émanant des établissements d'enseignement et de recherche français ou étrangers, des laboratoires publics ou privés. 


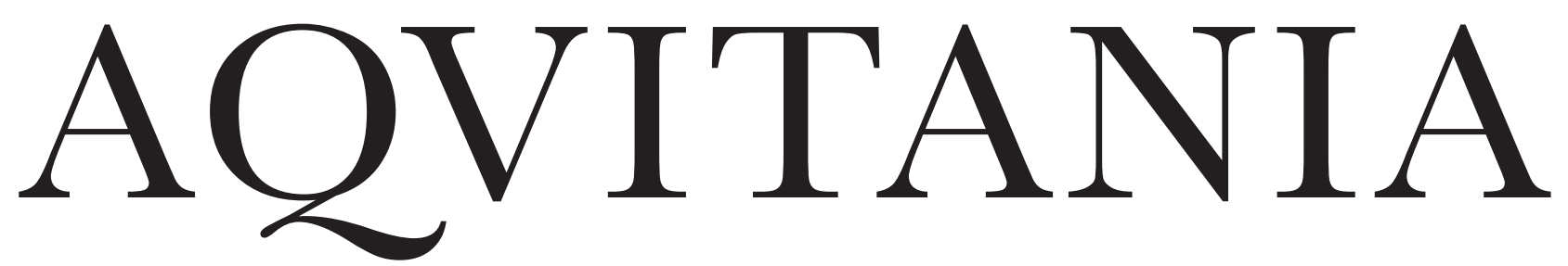

TOME 32

2016

\author{
Revue interrégionale d'archéologie \\ Aquitaine \\ Limousin \\ Midi-Pyrénées \\ Poitou-Charentes
}

Revue publiée par la Fédération Aquitania, avec le concours financier

du Ministère de la Culture, Direction du Patrimoine, Sous-Direction de l'Archéologie et de l'Université Bordeaux Montaigne,

et soutenue par l'Institut des Sciences Humaines et Sociales du CNRS 


\section{Couverture :}

- Paule, buste 3 (cl. A. Maillier, Bibracte nº 81 680).

- Col de l'amphore W. 51.

- Perle bleu cobalt, Bernorio 2012 (cl. Équipe Monte Bernorio, dessin A. Martínez Velasco, équipe Monte Bernorio). 


\section{Sommaire}

Auteurs

G. Seguin, K. Zipper, Le groupement d'enclos fossoyés de Bellevue à Châteaubernard (Charente)

J. F. Torres Martínez, A. Martínez Velasco, S. de Luis Mariño (Traduction : G. Cabanillas), Les perles en pâte de verre de l'oppidum de Monte Bernorio (Villarén, Palencia) et du nord de la péninsule Ibérique. Échanges et relations entre le nord de l'Espagne et le sud de la France à l'âge du Fer

C. MOREAU, La sculpture anthropomorphe en pierre du second âge du Fer dans l'Ouest de la Gaule

L. Borau, H. Gaillard, F. Riviėre, F. Sellami, Eau publique et eau privée à Vesunna. État de la question.

V. Elizagoyen, G. Hulin, C. Fondeville, V. Geneviève, V. Pasquet, S. Vigier, Une agglomération antique en Périgord: Les Olivoux à Montignac-sur-Vézère (Dordogne).

C. ChABRIÉ, La villa de Pardissous à Massels (Lotet-Garonne). Un exemple de petit établissement rural du milieu du ${ }^{\text {eq }}$ s. p.C...........

M. BROCHOT, La consommation du verre dans le quartier périphérique de l'agglomération secondaire antique de La Vayssière (L'Hospitalet-du-Larzac, Aveyron)

F. BeRTHAUlt, Les amphores de la nécropole paléochrétienne de Saint-Seurin à Bordeaux; réflexion sur le commerce des amphores dans le Sud-ouest au Bas-empire.

R. Barroso Cabrera, J. Carrobles Santos, J. Morín de Pablos, I. María Sánchez Ramos, Toletum. Ciuitas, suburbium, territorium. La construction d'une sedes regia wisigothique.

F. Boutoulle, É. JeAn-CourRet, Le complexe palatial alto-médiéval de Saint-Projet (Bordeaux, Gironde)......

J. Ollivier, C. Calmés, R. Carme, F. Dieulafait, C. Hallavant, J. Massendari, L. Pédoussaut, Toulouse, 16-18 rue des Pénitents Blancs: évolution d'un quartier oriental de la ville du Haut-Empire à nos jours.

\section{RECOMMANDATIONS AUX AUTEURS}




\section{Auteurs}

\author{
Rafael Barroso Cabrera \\ Frédéric Berthault \\ Laetitia Borau
}

Frédéric Boutoulle

Marion Brochot

Christophe Calmés

Rémi Carme

Jesús Carrobles Santos

Christophe Chabrié

Francis Dieulafait

Vanessa Elizagoyen

Carole Fondeville

Hervé Gaillard

Vincent Geneviève

Charlotte Hallavant

Guillaume Hulin

Ézéchiel Jean-Courret

Susana de Luis Mariño

Julie Massendari

Antxoka Martínez Velasco
Audema. Auditores de Energía y Medio Ambiente ; rbacab@gmail.com

Institut Ausonius - UMR 5607 ; frederic.berthault@orange.fr

Chargée de recherches au CNRS, Institut Ausonius - UMR 5607, LabEx Sciences Archéologiques de Bordeaux Université Bordeaux Montaigne ; laetitia.borau@u-bordeaux-montaigne.fr

Professeur en histoire du Moyen Âge, Institut Ausonius - UMR 5607, LabEx Sciences Archéologiques de Bordeaux Université Bordeaux Montaigne ; frederic.boutoulle@u-bordeaux-montaigne.fr

Membre associé TRACES (UMR 5608 CNRS/UT2J) ; marionbrochot@laposte.net

Archéologue et historien. Hadès, 9 rue Vidailhan, 31130 Balma - TRACES - UMR 5608 Université Toulouse Jean Jaurès ; christophe.calmes@hades-archeologie.com

Archéologue et céramologue. Hadès, 74 avenue du Midi, 63800 Cournon-d'Auvergne ; remi.carme@hadesarcheologie.com

Real Fundación Toledo ; jcarrobles@elgreco2014.com

Bénévole, Association des Archéologues du Lot-et-Garonne ; chabrie.christophe@neuf.fr

Numismate. Hadès, 9 rue Vidailhan, 31130 Balma - TRACES - UMR 5608 Université Toulouse Jean Jaurès ; francis.dieulafait@hades-archeologie.com

Chargée d'étude et de recherche, Inrap, chercheur associé à l'EA 3002 ITEM, coordinatrice du projet de recherche "Montignac Le Buy : bilan de dix ans d'archéologie préventive”, Responsable de l'opération archéologique programmée ; vanessa.elizagoyen@inrap.fr

Maquettiste, Inrap ; carole.fondeville@inrap.fr

Drac ALPC - SRA site de Bordeaux

Numismate, Inrap, chercheur associé à l'UMR 5060 IRAMAT-CEB ; vincent.genevieve@inrap.fr

Carpologue. Hadès, 9 rue Vidailhan, 31130 Balma - TRACES - UMR 5608 Université Toulouse Jean Jaurès ; charlotte.hallavant@hades-archeologie.com

Géophysicien, Inrap, chercheur associé à l'UMR 7619 METIS ; guillaume.hulin@inrap.fr

Maître de conférences en histoire du Moyen Âge, Institut Ausonius - UMR 5607, LabEx Sciences Archéologiques de Bordeaux Université Bordeaux Montaigne ; ezechiel.jean-courret@u-bordeauxmontaigne.fr

Departamento de Prehistoria Universidad Complutense de Madrid. Facultad de Geografía e Historia. Profesor Aranguren sn. 28040 Madrid. Instituto Monte Bernorio de Estudios de la Antigüedad del Cantábrico (IMBEAC). Colectivo para la ampliación de estudios de la Arqueología Prehistórica C.A.E.A.P. Investigadora Técnico Superior del Proyecto "Monte Bernorio en su entorno” ; susanadeluismarino@ gmail.com ; imbeac@gmail.com

Archéozoologue. Hadès, 9 rue Vidailhan, 31130 Balma ; julie.massendari@hades-archeologie.com

Instituto Monte Bernorio de Estudios de la Antigüedad del Cantábrico (IMBEAC). C/ Leira No 29, 20 Dcha. 28043 Madrid. Investigador Técnico Superior del Proyecto "Monte Bernorio en su entorno". ; antxokagaldakao@hotmail.com ; imbeac@gmail.com 
Chloé Moreau

Jorge Morín de Pablos

Julien Ollivier

Vincent Pasquet

Laëtitia Pédoussaut

Frédéric Rivière

Isabel María Sánchez Ramos

Guillaume Seguin

Farid Sellami

Jesús F. Torres Martínez

Serge Vigier

Katinka Zipper
Bibracte EPCC ; chloemoreau0804@gmail.com

Audema. Auditores de Energía y Medio Ambiente ; jmorin@audema.com

Archéologue. Hadès, 9 rue Vidailhan, 31130 Balma ; julien.ollivier@hades-archeologie.com

Topographe, Inrap ; vincent.pasquet@inrap.fr

Céramologue et étude du verre. Hadès, 9 rue Vidailhan, 31130 Balma - TRACES - UMR 5608 Université Toulouse Jean Jaurès ; laetitia.pedoussaut@hades-archeologie.com

TRACES UMR 5608 Université Toulouse Jean Jaurès

Institut Ausonius - UMR 5607, Université Bordeaux Montaigne ; isabel.sanchez@u-bordeaux-montaigne.fr

ÉVEHA POITIERS ; guillaume.seguin@eveha.fr

Inrap ; farid.sellami@inrap.fr

Departamento de Prehistoria Universidad Complutense de Madrid. Facultad de Geografía e Historia. Profesor Aranguren sn. 28040 Madrid. Instituto Monte Bernorio de Estudios de la Antigüedad del Cantábrico (IMBEAC). Investigador contratado Proyecto Forma Hispaniae Prerromanae. Ref. HAR2010-21650 (Subprograma HIST).

Director del Proyecto "Monte Bernorio en su entorno” ; ketxutorres@yahoo.com ; imbeac@gmail.com

Assistant d'étude et de recherche, Inrap ; serge.vigier@inrap.fr

ARCHEODUNUM -SAS - Agence Beuvray Centre Archéologique Européen ; k.zipper@archeodunum.fr 
Frédéric Boutoulle, Ézéchiel Jean-Courret

\section{Le complexe palatial alto-médiéval de Saint-Projet (Bordeaux, Gironde)}

\begin{abstract}
RÉSUMÉ
L'identification du complexe palatial de Saint-Projet, par l'analyse des textes et de la morphologie urbaine de Bordeaux, renouvelle l'historiographie du haut Moyen Âge, période encore perçue comme bien obscure. Jamais observé par les archéologues, cet ensemble forme une unité de plan constituée d'un tracé d'enceinte englobant une opération d'urbanisme ordonnancée autour de la place Saint-Projet ; la datation relative des formes urbaines associe cette unité au haut Moyen Âge. Bien que postérieurs, les textes des XI ${ }^{e}$-XIII ${ }^{\mathrm{e}} \mathrm{s}$. documentent cet ensemble au moment où le pouvoir l'abandonne progressivement au profit du nouveau pôle de l'Ombrière ; les composantes identifiées (palais de la Salle, chapelle palatiale, atelier monétaire, portes, puits), comparables à d'autres curtes, posent la question des origines de cet ensemble et de son rapport avec les incursions scandinaves du milieu du IX $\mathrm{x}^{\mathrm{s}}$.
\end{abstract}

\section{MOTS-CLÉS}

Bordeaux, palais, curtis, chapelle palatiale, atelier monétaire, morphologie urbaine, unité de plan, tracé d'enceinte, opération d'urbanisme

\begin{abstract}
Revealed by the analysis of texts and urban morphology, the palatial complex of Saint-Projet renews the historiography of the early Middle ages which is still considered as a dark period. Never observed by archeology, this set forms a plan unit which is constituted by a urban fortification trace includind a town planning organized around Saint-Projet place ; relative chronology of urban forms associates this set to early Middle ages. The subsequent texts of the $\mathrm{XI}^{\text {th }}$-XIII ${ }^{\text {th }}$ centuries tell how the power give up gradually this site in favor of a new power pole (Ombrière), and also the components of Saint-Projet complex (palace named La Salle, palatial chapel, mint, gates, well). These elements, similar to others curtes, ask when they were build and its relations with Scandinavian invasions.
\end{abstract}

\section{KEYWORDS}

Bordeaux, palace, curtis, palatial chapel, mint, urban morphology, plan unit, urban fortification trace, town planning. 
L'étude des fortifications et résidences élitaires médiévales a été renouvelée depuis une quinzaine d'années du fait de la multiplication des découvertes archéologiques, de la relecture des sources écrites et de la volonté des chercheurs d'aborder cette question de manière interdisciplinaire en intégrant à leurs pratiques des approches jusque-là marginalisées (morphologie parcellaire). L'inventaire réalisé entre Loire et Pyrénées au colloque de Pau (2002) dresse un panorama pour les Xe $\mathrm{XV}^{\mathrm{e}} \mathrm{s} .{ }^{1}$; pour les périodes hautes, le projet collectif de recherche lancé par Luc Bourgeois en 2008 a établi et réuni les monographies de quelques 200 sites entre Loire et Garonne et a abouti à une stimulante synthèse lors du colloque de Chauvigny ${ }^{2}$. Le corpus ${ }^{3}$ révèle l'abondance et la variété typologique de l'habitat aristocratique (palais carolingiens, palais ducaux et comtaux ou épiscopaux, résidences rurales, curtes) même si quelques sites ont été écartés du fait de la nature, peut-être trop ténue, des indices permettant de les identifier de façon certaine. Tel est le cas du complexe palatial de Saint-Projet à Bordeaux sur lequel n'ont été esquissées jusqu'à présent que des approches partielles qu'il convient de croiser.

La découverte, au coeur de la cité antique de Bordeaux, d'un ensemble palatial du haut Moyen Âge lié à une enceinte jusque-là inconnue, est assez récente. La mise au jour de ces données par l'analyse des textes et de la morphologie parcellaire, renouvelle très largement les connaissances sur l'histoire de la ville pendant le haut Moyen Âge où pendant longtemps tout semblait avoir été dit depuis la monumentale Histoire de Bordeaux de C. Higounet ${ }^{4}$. En 2003, le palais de Saint-Projet était présenté dans un article de la Revue archéologique de Bordeaux 5 . Depuis cette date, les travaux sur la fabrique urbaine de Bordeaux ont apporté de nouveaux éléments, permettant de mieux comprendre l'environnement de l'ensemble palatial et de proposer de nouvelles interprétations à quelques pièces du dossier textuel analysé en $2003^{6}$. Le présent article fera donc le point de ce que l'on sait sur cet ensemble, par les textes, les représentations icono-cartographiques et par l'analyse morphologique car les sondages archéologiques n'ont pour l'heure livrés aucune structure se rapportant à ce pôle de pouvoir?

\section{Morphologie du pÔle de SAINT-Projet}

\section{Source et méthode}

Source spatiale de référence, le cadastre de 1810-1821 livre un état parcellaire ancien, précis et fiable, antérieur aux considérables transformations de l'haussmannisation bordelaise ${ }^{8}$. À hauteur de la place SaintProjet, ce plan cadastral garde la mémoire des tracés anciens de la rue Sainte-Catherine dont l'alignement

\footnotetext{
1. Barraud et al. 2005.

2. Bourgeois \& Rémy 2014.

3. Bourgeois 2008, 109-118 (notice sur Saint-Projet).

4- Higounet 1963.

5. Boutoulle 2003.

6- Jean-Courret 2006, 298, 325 et 355; Lavaud \& Jean-Courret 2009, t. 1, pl. 2 ; t. 2, 49-50 ; t. 3, 76-77.

7. Les premières observations archéologiques réalisées au n ${ }^{\circ} 2$ de la place Saint-Projet remontent à 1850 : lors de la destruction d'une partie de l'église pour construire "la maison qui se trouve au couchant de la fontaine", des sépultures médiévales sont exhumées (Sansas 1861). Peu après, en 1861, on découvre de nombreux matériaux d'époque antique (débris de tesselles, tessons de céramiques, remblais constitués de mortiers très indurés), parmi lesquels un élément de colonne en marbre portant une dédicace à Mercure (Sansas 1861 ; Jullian 1887-1890, t. 1, n 11, 37-38). En 1933, une fouille limitée, réalisée (sur la place?) dans le cadre de l'installation de toilettes publiques souterraines, met au jour un "chapiteau en marbre gris gothique" (Ricaud 1933). En 1955-1956, plusieurs sépultures médiévales sont découvertes sur les côtés sud et ouest de l'église Saint-Projet et sont associées au cimetière paroissial de la fin du Moyen Âge (Nony \& Redeuil 1955-1956). Sur la base des vestiges antiques découverts au XIX⿸广 s., A. Maillé a émis l'hypothèse qu'un premier édifice de culte chrétien avait été fondé sur un temple dédié à Mercure (Maillé 1960, 180-181 et 230). L'analyse archéologique du bâti effectuée par A. Marin en 2001 a permis d'étudier toute l'élévation de l'église médiévale : dans les caves modernes du 97 rue Sainte-Catherine, l'archéologue a relevé quelques substructions gallo-romaines associées aux I I $\mathrm{I}^{\mathrm{e}} \mathrm{V}^{\mathrm{e}} \mathrm{s}$. L'homogénéité des élévations médiévales témoigne d'un "édifice ayant disposé d'une vaste programme de reconstruction au XIV ${ }^{e}$ s., probablement à l'emplacement d'un édifice plus ancien dont on ignore tout aujourd'hui” (Marin 2001a, 23 ; Marin 2001b). Sur ce secteur, les niveaux du haut Moyen Âge semblent donc avoir été complètement arasés ; les dernières observations réalisées en 2009 -2010 pour le réaménagement de surface de la place Saint-Projet et des rues adjacentes n'ont rien livré et n'ont pas donné lieu à un rapport. Les îlots qui confrontent le nord de la place n'ont pas fait l'objet d'observations archéologiques.

8- Jean-Courret 2006a, t. 2 pour le protocole d'édition du plan.
} 
est en cours quelques décennies après, comme l'atteste le cadastre de 1850-1855'.

La figure 1 situe quelques éléments topographiques sur le squelette urbain de la ville du Premier Empire : repères actuels de l'esplanade des Quinconces, du Pont-depierre, de la gare et de la cathédrale ; données relatives au modelé du site (terrassesalluvialesetréseau hydrographique urbain) ; enfin, les unités topographiques fortifiées soulignées par quatre tracés d'enceinte ${ }^{10}$.

L'analyse en plan des processus de fabrique spatiale ${ }^{11}$ est le fruit d'un long apprivoisement du parcellaire bordelais ; la démarche a été menée indépendamment des autres données disponibles. Elle repose sur le potentiel historique de la mémoire du plan dont l'étude régressive livre des faits morphologiques à l'état de traces, souvent non documentés par ailleurs. L'analyse morphologique relève d'approches conjointes, l'une, descriptive vise à examiner les composantes et les comportements de la trame (voirie, parcellaire, bâti) dans leur rapport au site, l'autre, hypothéticodéductive, consiste à identifier des unités de plan $^{12}$ et à formuler, à partir d'elles, des hypothèses urbanistiques. Une chronologie

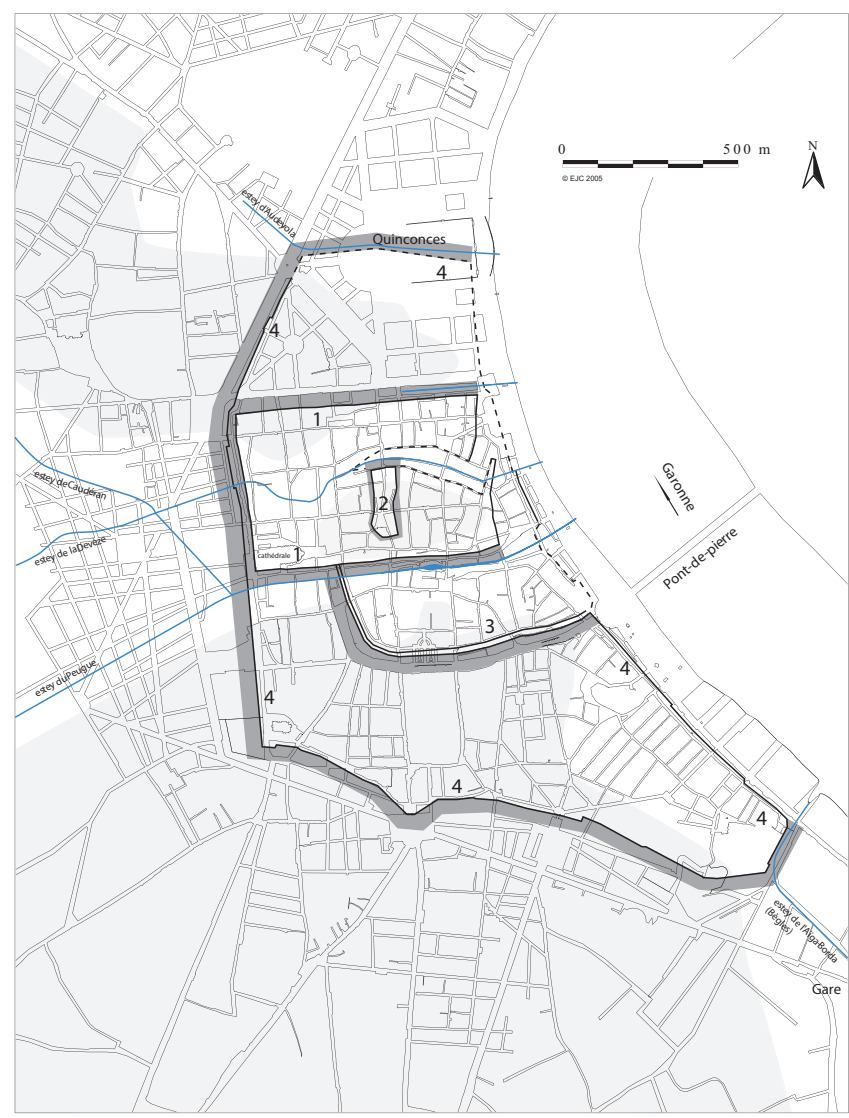

Fig. 1. Hypothèses de tracés d'enceintes, zone de non aedificandi et fossés (fond de plan : cadastre de 1810-1821, cartographie É. JeanCourret). relative d'élaboration des formes découle de l'examen de la disposition interne et relative des unités identifiées. La morphologie des aménagements de Saint-Projet est décrite ci-après avant d'être repositionnée au regard des autres faits identifiés en plan.

\section{L'aménagement urbain combiné de Saint-Projet}

En position centrale au cœur de cité, remparée depuis la fin du III s. (fig. 1, tracé 1), le secteur de SaintProjet a fait l'objet d'un aménagement urbain combiné c'est-à-dire un "groupe d'aménagements dont les réalisations successives sont organisées suivant une composition unitaire" ${ }^{13}$. L'analyse morphologique identifie, à l'état de trace, trois aménagements parfaitement imbriqués : le premier comporte toutes les

9. Lavaud \& Jean-Courret 2009, t. 1, pl. 02.

10- Jean-Courret 2006a, 296-300 pour l'analyse de chacun de ces tracés d'enceinte.

11- La méthode d'analyse a déjà été exposée en détail dans Aquitania à propos du cas dacquois (Jean-Courret 2010, 160-164).

12. Gauthiez 2003, 194 : "partie d'un plan d'agglomération présentant des caractères géométriques propres en termes de voirie, de parcellaire et de bâti, avec une unité géométrique interne la distinguant de son environnement et permettant de lui attribuer une genèse particulière".

13. Ibid., 57. 


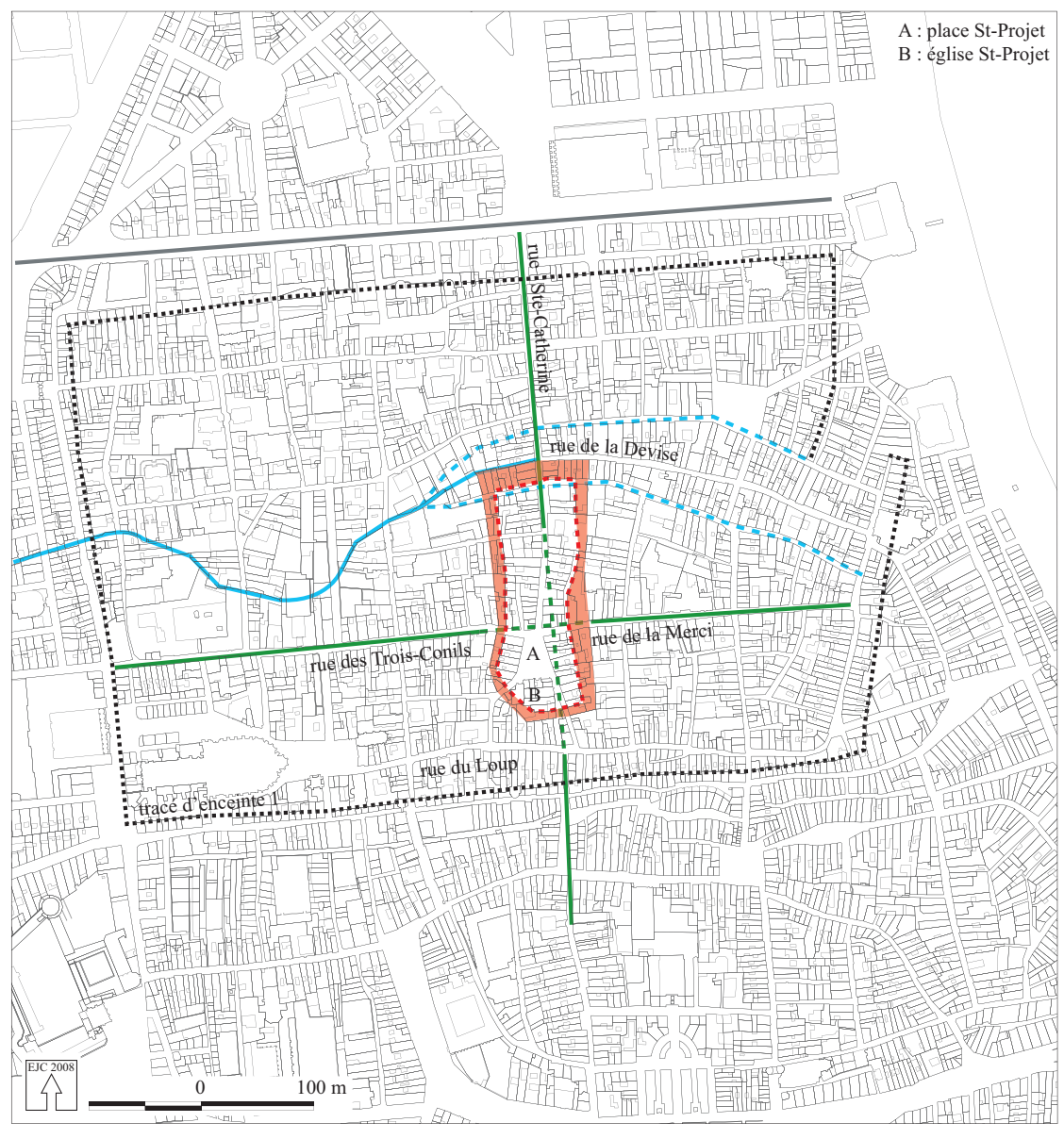

Fig. 2. Le tracé d'enceinte de Saint-Projet (cartographie É. Jean-Courret).

caractéristiques liées à un tracé d'enceinte ; le deuxième correspond à une opération d'urbanisme dont l'emprise ne dépasse pas la portion d'espace enclose ; le troisième comprend les aménagements viaires de liaison et de contournement résultant du tracé d'enceinte.

\section{Le tracé d'enceinte (fig. 1, tracé 2 et fig. 2)}

Si l'on ne peut identifier la nature matérielle d'une enceinte par les traces qu'elle laisse en plan (levée de terre, palissade en bois, rempart en pierre, continuum bâti formant enceinte), un tracé d'enceinte est morphologiquement caractérisé comme une forme englobante et continue, une ceinture autonome, parfois ouverte, mais qui profite alors d'une défense antérieure ou d'un élément défensif naturel ${ }^{14}$. Il est repérable sous la forme d'au moins deux alignements parallèles et emboîtés formant ceinture et distants, au plus, de quelques dizaines de mètres : l'alignement interne garde mémoire de l'élévation de la fortification, l'alignement externe matérialise l'existence de fossés ou d'une zone de non aedificandi. La réalisation d'une enceinte génère des modifications importantes de la voirie : interruption de voie, contournement, patte 


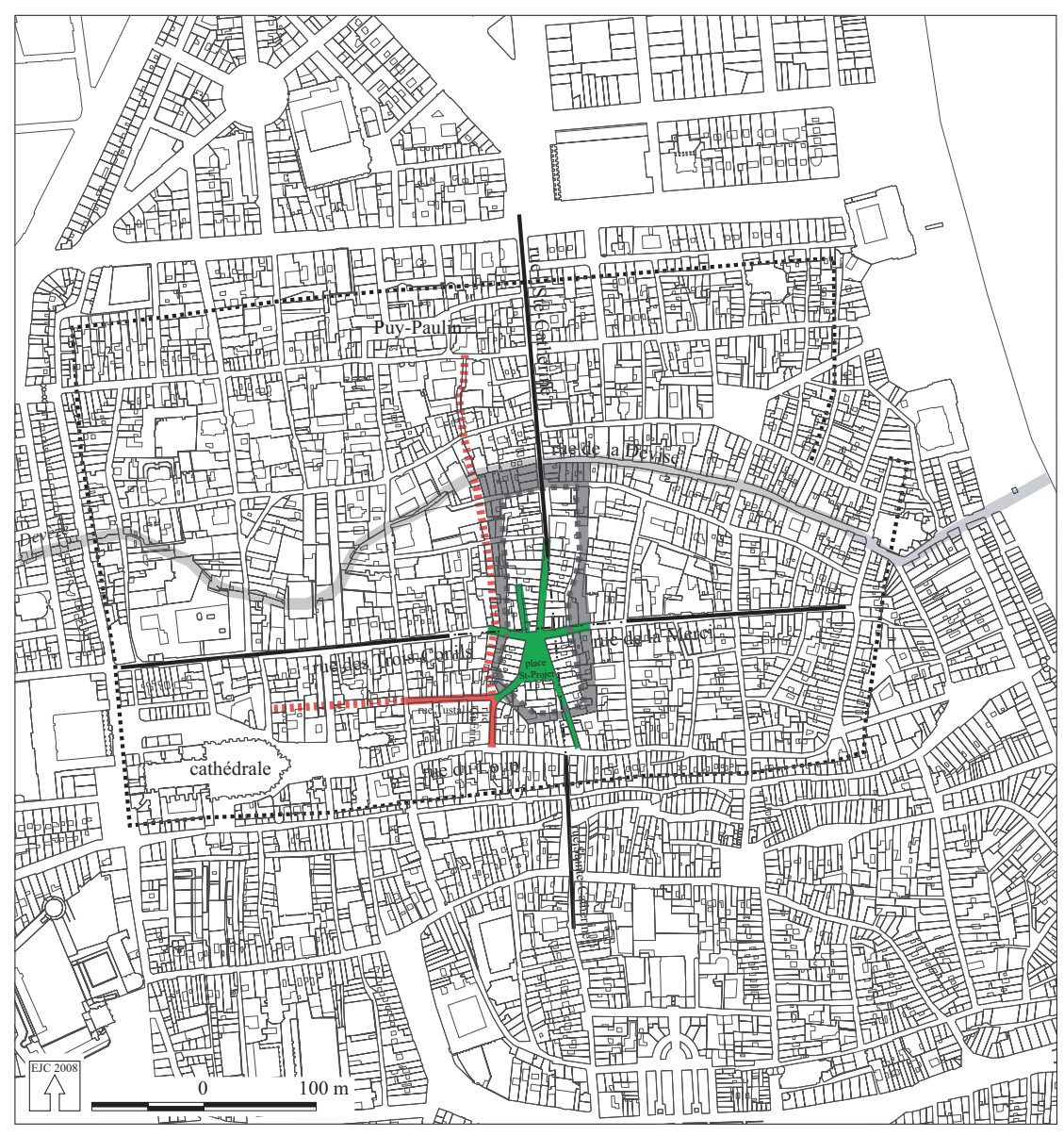

Fig. 3. Les opérations d'urbanisme connectées au tracé d'enceinte de Saint-Projet (cartographie É. Jean-Courret).

d'oie pour accéder à une porte, induction par effet de paroi ${ }^{15}$ de système d'escarpe et de contrescarpe, caractéristiques toutes observables à Saint-Projet.

Un alignement remarquable ceinture la place Saint-Projet entre la Devèze (rue de la Devise) et la rue du Loup (fig. 2). Il forme un polygone allongé aux contours sinueux centré sur le carrefour des rues SainteCatherine (nord/sud), des Trois-Conils et de la Merci (est-ouest), polarisées par la place Saint-Projet, de forme trapézoïdale. Cet alignement est accompagné d'une frange de 20/25 m de large en moyenne qui pourrait constituer un fossé ou une zone de non ædificandi autour de l'enceinte. L'enveloppe, repérable sous la forme de deux alignements parallèles et emboîtés formant ceinture, couvre une superficie de 2 ha dont 1,4 ha pour l'espace enclos. Il génère des comportements viaires extrêmement contrastés de part et d'autre de son tracé. L'orientation des rues comprises à l'intérieur de cet alignement dévie profondément de l'axe qu'elles ont à l'extérieur de la structure. Au sud-ouest, les rues Guérin et Tustal forment une patte d'oie suggérant l'emplacement d'une porte et permettant de relier cette unité au reste du tissu urbain.

15- Ibid., 342 : "localisation et disposition d'édifices ou d'aménagements urbains induites par la présence d'un équipement ou d'un aménagement de l'espace allongé formant barrière". 


\section{L'opération d'urbanisme interne au tracé d'enceinte (fig. 3)}

À l'intérieur du tracé d'enceinte, la place Saint-Projet forme un trapèze équilibré (composition symétrique sur un axe nord-sud) aux angles duquel viennent se connecter plusieurs voies. La place se love à l'angle sudouest du carrefour formé par le cardo Saint-Catherine et la rue des Trois-Conils. Ces deux axes semblent avoir eu un tracé originel rectiligne que l'opération a bouleversé. La distribution des voies, réparties aux quatre angles de la place (quatre voies sur les deux pointes nord, deux au sud) souligne une réalisation contemporaine des différentes composantes de l'aménagement intimement lié au tracé d'enceinte. La forme trapézoïdale très régulière implique une composition monumentale forte avec, au sud, l'église Saint-Projet, et au nord, un ensemble que l'analyse ne permet pas de distinguer. Au centre, la place Saint-Projet pourrait être un espace commercial (marché) implanté non loin du bassin portuaire de la Devèze.

\section{Le système viaire de liaison et de contournement (fig. 3)}

À l'extérieur du tracé d'enceinte, l'enveloppe défensive de Saint-Projet a nécessité la mise en place de voies de liaison et de contournement. On peut identifier ce réseau, sur la face occidentale, par l'intermédiaire de la patte d'oie formée à l'angle sud-ouest du tracé d'enceinte, indiquant la position d'une porte. Un alignement prolonge la rue Tustal à l'ouest, en direction de la cathédrale Saint-André. Au sud enfin, la rue Guérin fait la liaison entre Saint-Projet et la rue du Loup qui est antérieure. Un alignement continu de parcelles et de limites parcellaires peut être interprété comme une trace de voirie, orientée nord-sud (pointillés) et reliant Saint-Projet au secteur de Puy-Paulin. L'ensemble des opérations de voirie couvre une surface de 1,5 ha.

L'imbrication profonde et l'ajustement de chacune des composantes (tracé d'enceinte, opération d'urbanisme interne, système viaire externe) font de cet ensemble un véritable aménagement combiné, certainement réalisé de façon synchrone. La disparition de la ligne de défense et des voies de contrescarpe pourrait également être simultanée. La superficie notoire de l'espace urbain enclos (1,4 ha) implique, au-delà des infrastructures viaires et monumentales qui le composent, l'englobement d'une part importante d'espaces privés probablement en lien avec les fonctions de la place.

\section{Chronologie relative des opérations}

Si l'on ne peut présenter ici en détail l'ensemble des trois autres principaux tracés d'enceintes, des cinq autres opérations d'urbanisme et des six lotissements identifiés et attribuables à la période qui courre de la fin $\mathrm{du} \mathrm{III}^{\mathrm{e}} \mathrm{s}$. au début du XVI $\mathrm{X}^{\mathrm{e}}$ s, il convient néanmoins de décrire les principaux mécanismes des rapports spatiaux exercés entre les aménagements afin d'établir une chronologie relative permettant de savoir à quelle phase appartiennent les aménagements de Saint-Projet (fig. 5).

L'assemblage des unités morphologiques fortifiées et de leur voirie attenante fournit un premier jalon (fig. 1). Dans le classique panorama des fortifications bordelaises, la position du complexe de Saint-Projet pose question (fig. 1, tracé 2). L'historiographie des processus de formation urbaine conçoit habituellement qu'une agglomération peut être fortifiée par plusieurs enceintes successives, concentriques ou juxtaposées, protégeant chacune un quartier ou un faubourg nouveau. Les circonstances qui président à l'érection de fortification sont diverses : soit une nécessité de défendre, et l'on a alors tendance à limiter au plus serré le périmètre protégé (c'est partiellement le cas du tracé 3 : bourg Saint-Eloi), soit une volonté d'extension urbaine et l'on protège alors une zone non urbanisée afin d'encourager son urbanisation (c'est le cas de l'enceinte de Philippe-Auguste à Paris, mais aussi fréquemment le cas des enceintes de réunion qui englobent les unités fortifiées préexistantes, les nouveaux faubourgs et même des secteurs rurbains, comme ici le tracé 4 de l'enceinte de la ville). À ces concepts s'ajoutent souvent celui de la distance au centre pour le Moyen Âge : chaque nouvelle unité fortifiée se greffe à l'extérieur d'unités préexistantes. 


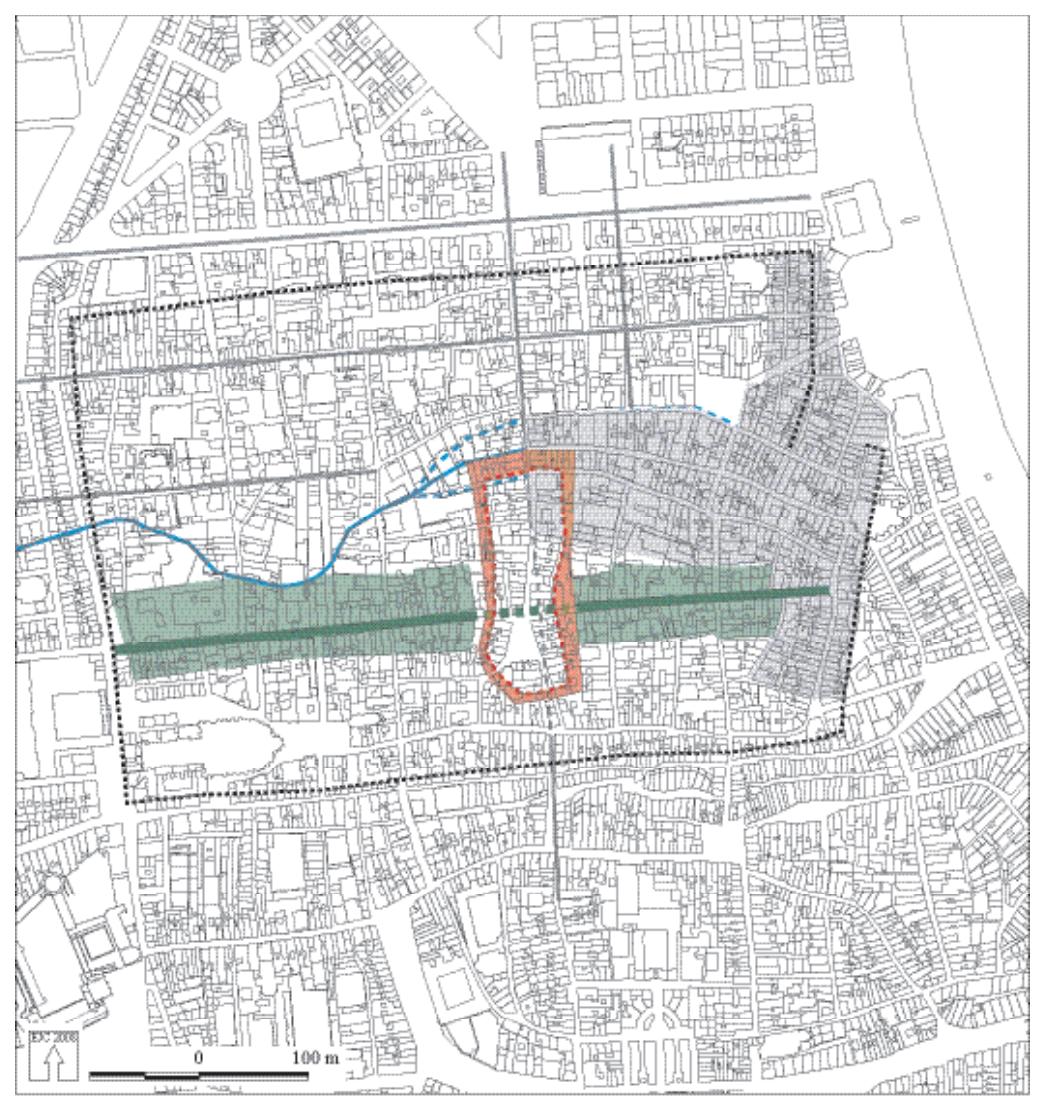

Fig. 4. Les opérations recoupées par le pôle de Saint-Projet (cartographie É. Jean-Courret).

Or, plusieurs indices morphologiques concluent à la mise en abîme du complexe de Saint-Projet. Les voies de contournement et de connexion repérées à l'ouest et au sud ont pour vocation de connecter le pôle au système viaire préexistant fondé sur une trame orthogonale établie durant les premières décennies du $\mathrm{I}^{\mathrm{er}} \mathrm{s}$. Cette trame, qui dépasse largement le tracé de l'enceinte antique (tracé 1), est celle qui structure la ville du Haut-Empire avant sa rétraction simultanée ou consécutive à l'érection du rempart à la fin du $\mathrm{III}^{\mathrm{e}} \mathrm{s}$. Ces deux éléments permettent de comprendre que le tracé de Saint-Projet est postérieur à la cité remparée. Sa faible emprise ( 2 ha) et sa position stratégique au centre de la ville permettent de supposer qu'il s'agit d'une structure née d'un souci - si ce n'est défensif au moins de polarisation, d'une volonté de créer un nouveau marqueur paysager, un nouveau centre de gravité ${ }^{16}$ et non d'une volonté d'expansion que l'on peut identifier partiellement pour la double courtine du bourg Saint-Éloi (tracé 3) et clairement pour l'enceinte de réunion de la ville (tracé 4) qui lui sont postérieures. Il pallie la carence défensive de l'enceinte antique qui présente une large ouverture à l'emplacement du port intérieur établi sur l'embouchure de la Devèze.

L'aménagement urbain combiné recoupe également deux autres opérations (fig. 4). Il empiète sur le secteur situé sur l'embouchure de la Devèze, marqué par la concentration d'une orientation de 19,8 (fig. 4 , gris ${ }^{17}$. Ce secteur est caractérisé par la reparcellisation d'un double peigne viaire distribuant le port intérieur

16- Jean-Courret 2014, 403-408.

17. Jean-Courret 2006a, 230 et 320-321. 


\begin{tabular}{|c|c|c|c|}
\hline 1 & 2 & 3 & 4 \\
\hline Phases & $\begin{array}{c}\text { Nature de } \\
\text { l'aménagement } \\
\text { Désignation et datation* }\end{array}$ & $\begin{array}{l}\text { Dispositions respectives permettant } \\
\text { d'établir un lien chronologique }\end{array}$ & Commentaires \\
\hline 1 & $\begin{array}{l}\text { Trame orthogonale } \\
\text { antique } \\
\text { époque augustéenne, } \\
\text { premières décennies } d u I^{\text {er }} s .\end{array}$ & - & $\begin{array}{l}\text { Mise en place d'une trame viaire } \\
\text { globalement orthonormée et connexion } \\
\text { avec les grandes voies de communication } \\
\text { terrestre (nord, ouest et sud) }\end{array}$ \\
\hline 2 & $\begin{array}{l}\text { Tracé d'enceinte } 1 \\
\text { enceinte de la cité } \\
\text { fin } d u \text { III's. }\end{array}$ & $\begin{array}{l}\text { Enceinte } 1 \text { centrée sur le port intérieur } \\
\text { antique et reprenant l'orientation de la } \\
\text { trame orthogonale }\end{array}$ & $\begin{array}{l}\text { Suggère une phase de rétraction des } \\
\text { secteurs urbains extra-muros et donc une } \\
\text { disparition partielle de la trame } \\
\text { orthogonale antique }\end{array}$ \\
\hline 3 & $\begin{array}{l}\text { Opération d'urbanisme } 1 \\
\text { reparcellisation progressive } \\
d u \text { port de la Devèze et } \\
\text { peignes viaires adjacents, } \\
\text { à partir } d u V^{\circ} \text { s. }\end{array}$ & $\begin{array}{l}\text { Dépasse l'emplacement du port intérieur } \\
\text { antique, axée sur le cours de la Devèze }\end{array}$ & $\begin{array}{l}\text { Suggère l'envasement du port et une } \\
\text { occupation agencée sur l'axe Devèze/ } \\
\text { Saint-Pierre }\end{array}$ \\
\hline 4 & $\begin{array}{l}\text { Opération d'urbanisme } 2 \\
\text { axe de liaison de Saint- } \\
\text { André à Saint-Pierre, } \\
\text { c. VII -VII's. }\end{array}$ & $\begin{array}{l}\text { Ne dépasse pas le cadre de l'enceinte } 1 \text {, ne } \\
\text { reprend pas le module de la trame } \\
\text { orthogonale, l'opération } 2 \text { est donc } \\
\text { postérieure. Elle recoupe également } \\
\text { 'opération } 1 \text {. }\end{array}$ & $\begin{array}{l}\text { Le rôle morphogène de l'orientation } \\
\text { viaire antique explique l'isoclinie logique } \\
\text { de cet axe de liaison entre le pôle } \\
\text { cathédral et Saint-Pierre. }\end{array}$ \\
\hline 5 & $\begin{array}{l}\text { Tracé d'enceinte } 2 \text { et } \\
\text { opération d'urbanisme } \\
\text { aménagement combiné } \\
\text { de Saint-Projet }\end{array}$ & $\begin{array}{l}\text { Engendre une modification de } \\
\text { l'orientation de la trame orthogonale } \\
\text { antique et de l'opération } 2\end{array}$ & $\begin{array}{l}\text { Système de défense interne ayant pour } \\
\text { objectif de protéger le pôle de Saint- } \\
\text { Projet et de pallier la carence de défense } \\
\text { à l'emplacement de l'opération } 1 \text {, sur le } \\
\text { port intérieur antique. }\end{array}$ \\
\hline 6 & $\begin{array}{l}\text { Tracé d'enceinte } 3 \\
\text { enceinte du bourg } \\
\text { Saint-Eloi, } \\
\text { première moitié du XIII's. }\end{array}$ & $\begin{array}{l}\text { L'enceinte } 3 \text { englobe l'opération } 4 \text { et } \\
\text { oblitère les lotissements } 1 \text { et } 2 \text {. } \\
\text { L'opération } 5 \text { semble contemporaine, elle } \\
\text { remodèle la partie nord du lotissement } 1 \\
\text { inséré dans l'enceinte } 3 \text {, en rapport avec } \\
\text { le castrum de l'Ombrière. }\end{array}$ & $\begin{array}{l}\text { Défense du pôle marchand de l'opération } \\
4 \text {, enceinte de défense ne prenant pas en } \\
\text { compte tous les faubourgs antérieurs } \\
\text { (lotissements } 1 \text { et } 2 \text { ), enceinte de prestige } \\
\text { marquée par une double ligne de } \\
\text { courtines et de fossés. }\end{array}$ \\
\hline 7 & $\begin{array}{l}\text { Tracé d'enceinte } 4 \\
\text { enceinte de la ville } \\
\text { (enceinte de réunion), } \\
\text { XIVes. - milieu XVI } \mathrm{X}^{e} \text { s. }\end{array}$ & $\begin{array}{l}\text { Enceinte d'extension dont le tracé suit } \\
\text { celui des grands lotissements et intègre } \\
\text { également des espaces rurbains. Postérieur } \\
\text { à toutes les opérations précédentes. }\end{array}$ & $\begin{array}{l}\text { Enceinte de réunion englobant les } \\
\text { fortifications antérieures }(1,2 \text { et } 3) \text {, les } \\
\text { édifices religieux et les faubourgs } \\
\text { (lotissements } 2,3,4,5 \text { et } 6 \text { ) }\end{array}$ \\
\hline
\end{tabular}

* Pour plus de clarté, ces deux informations ont été ajoutées à la colonne 2 par rapport à la synthèse exhaustive des opérations fournies dans Jean-Courret 2006, 344.

fig. 5. Chronologie relative des tracés d'enceintes 1 à 4 et des aménagements urbains recoupés par l'aménagement combiné de Saint-Projet.

antique suite à l'envasement de la Devèze, phénomènes liés et très progressifs, qui s'étalent du $\mathrm{VI}^{\mathrm{e}} \mathrm{s}$. au X $\mathrm{X}^{\mathrm{e}} \mathrm{s}$. sans obstruer de façon soudaine la vocation portuaire de l'estey ${ }^{18}$. La seconde concerne la liaison de SaintAndré à Saint-Pierre soit l'axe viaire composé des rues des Trois-Conils, de la Merci, Saint-Siméon et Maucoudinat (fig. 4, vert). Cette opération, bien qu'isocline à la trame antique, ne déborde pas le tracé d'enceinte 1 à l'instar des autres decumani ; elle génère un module d'insulae nettement plus allongé 
$(120 \mathrm{~m}$ x $150 \mathrm{~m})$ que le module repéré dans la portion septentrionale de la trame $(120 \mathrm{~m}$ x $120 \mathrm{~m}$, en moyenne). Elle ne semble donc pas appartenir à la trame viaire antique et apparaît donc comme postérieure à elle, ce que corroborent les observations archéologiques menées sur un tronçon de voirie significatif de la rue des Trois-Conils ${ }^{19}$. Cet axe peut également être interprété comme une structuration interne de la cité remparée ayant pour objectif de faciliter les liaisons entre le pôle cathédral de Saint-André (sud-ouest) et le secteur fluvial autour de l'église Saint-Pierre. La régularité de la voie et du parcellaire qui lui est associé pourrait indiquer que la voie est percée en une traite. Parce qu'ils oblitèrent une partie de ces opérations (rupture d'orientation du cardo Sainte-Catherine et de l'axe des Trois-Conils au profit de la composition urbaine de la place, empiétement sur la portion sud-ouest du secteur de reparcellisation de la Devèze), les aménagements de Saint-Projet, semblent donc être postérieurs à elles.

Du point de vue morphologique, il est impossible d'affiner plus la datation de l'ensemble des aménagements de Saint-Projet (fig. 5). Avec les précautions qu'il convient de prendre en l'absence presque total de données concernant la période couvrant les $\mathrm{VII}^{\mathrm{e}} \mathrm{x} \mathrm{x}^{\mathrm{e}} \mathrm{s}$., et eu égard à la connaissance lacunaire que l'on a de la ville à cette époque, on peut néanmoins se risquer à avancer quelques pistes. Avec les opérations concernantla reparcellisation de l'embouchure de la Devèze et l'axe de liaison Saint-André / Saint-Pierre, le complexe de Saint-Projet témoigne d'actions urbanistiques d'une période que l'historiographie avait jusqu'à présent cantonné à l'obscurité, à la régression, au péril des invasions. L'espace urbain du Bordeaux du haut Moyen Âge, bien que cantonné dans les limites de la cité remparée, apparaît au contraire comme un territoire réapproprié par la société urbaine, à commencer par ses élites dirigeantes. Alors que l'Église bordelaise semble tenir l'autorité et le pouvoir entre le IV e s. et le VII s., Saint-Projet traduit, comme on va le voir, l'affirmation du pouvoir laïc et sa volonté de sédentariser un certain nombre de prérogatives (résidentielles et curiales), d'exercer son contrôle sur les espaces économiques (marché) en plus de ses fonctions défensives entre les $\mathrm{VII}^{\mathrm{e}} \mathrm{s}$. et $\mathrm{X}^{\mathrm{e}} \mathrm{s}$. Le retour du monnayage bordelais au VII ${ }^{e}$ s. et les liens probables tissés entre les détenteurs de l'autorité jusqu'au Moyen Âge central en attestent. Les rares textes du Moyen Âge présentés ci-après, bien que très postérieurs, documentent certains équipements de cet ensemble palatial, appelé La Salle au XI ${ }^{\mathrm{e}}$ s., et dont l'existence parait probable antérieurement.

\section{L'ensemble palatial de La Salle à Saint-Projet vu par les textes}

Le palais de La Salle, situé à Saint-Projet, apparaît pour la seule et unique fois dans une donation datée du 5 mai 1070 du duc d'Aquitaine Guy Geoffroy, comte de Bordeaux depuis 1040 et duc de Gascogne depuis moins de temps, en faveur de l'abbaye de Saint-Sever ${ }^{20}$. Le texte fait partie du fonds de Saint-SeverCap-de-Gascogne, la grande abbaye bénédictine du diocèse d'Aire, fondée en 988. L'abbaye possède alors l'église Saint-Germain située dans le suburbium nord de Bordeaux et un ensemble foncier au sud de la ville ${ }^{21}$. Guy Geoffroy commence par confirmer les donations de ses prédécesseurs, Guilhem Sanche, Bernard et Sanche Guilhem en faveur de Saint-Sever. À la suite de quoi, il cède à l'abbaye gasconne :

“intégralement, loyalement et sans aucune réserve sur l'exemption de cens, le palais de Bordeaux, situé en avant [ou devant] des portes de Saint-Projet martyr (prae foribus S. Projecti), et que le peuple appelle La Salle, avec son puits, son atrium, des chambres (cubiculis) et des dépendances (appendiciis) faisant juridiquement partie de ce palais, au Seigneur Dieu et au prince des apôtres Pierre, ainsi qu'à l'illustre martyr Sever"22.

19. Gaidon-Bunuel 2002.

20. Pon \& Cabanot, éd. 2010, $n^{\circ} 22$.

21- Ibid., $\mathrm{n}^{\circ} 7$ et $\mathrm{n}^{\circ} 41(1028-1072)$.

22- Ibid., $\mathrm{n}^{\circ} 22$ : tradens insuper, ut dux et comes totius Aquitaniae et Gasconiae, pro animae meae remedio (...) palatium Burdegalae ab omni censu liberum et immune, quod prae foribus S. Projecti martyris habetur, quodque vulgares Salam vocant, Domino Deo et apostolorum principi Petro, necnon et egregio martyri Severo, integre et sincere absque ullius census retractione, cum puteo et atrio, cubiculis et appendiciis jure huic palatio competentibus, donoque perenniter delego. 
Ce palais dont il n'a été question dans aucun autre texte avant celui-ci, apparaît en pleine lumière au moment où le duc s'en débarrasse et où il procède à une forme de démantèlement. En effet, si Saint-Sever reçoit les chambres, le puits et l'atrium, en revanche la salle qui a donné son nom au palais ne fait pas partie de la donation. Quelques années plus tard, en 1072 ou en 1077, c'est un autre établissement religieux, le prieuré Saint-Martin du Mont-Judaïque, dépendant de Maillezais, qui reçoit du même duc la capella palatii de Bordeaux ${ }^{23}$. En deux donations situées à deux ou sept ans d'écart, nous retrouvons les trois éléments caractéristiques des palais mais dorénavant séparés : une aula à la destinée (pour l'instant) inconnue, un ensemble résidentiel abandonné à Saint-Sever et la capella donnée à Saint-Martin du Mont-Judaïque.

Cependant, le désengagement de la puissance ducale de Saint-Projet a commencé plus tôt. On sait par une confirmation du duc d'Aquitaine Guilhem IX, que ses prédécesseurs, les ducs Sanche Guilhem (10101032), Eudes (1032-1040) et son père Guy Geoffroy, avaient donné à la cathédrale Saint-André le tiers de l'atelier monétaire, c'est-à-dire le tiers de ses revenus (tertiam partem nostre camere seu monete) ${ }^{24}$. Or, comme nous allons le voir, cet atelier monétaire était situé entre la Devèze et Saint-Projet. Le démantèlement de l'ancien palais par Gui Geoffroy ne fait donc que consacrer un mouvement commencé plus tôt, certainement motivé par le désir de ne pas tout abandonner au même bénéficiaire.

Ce démantèlement doit être mis en relation avec l'apparition, au même moment dans les textes, de la tour où le duc réside à Bordeaux ${ }^{25}$. Cette tour de $18 \mathrm{~m}$ sur $14 \mathrm{~m}$, ultérieurement appelée l'Arbalesteyre, commence en effet à être citée en 1080. Comme Jacques Gardelles l'a reconnu, elle appartient à la famille des tours maîtresses aquitaines de cette période. Tout indique que Guy Geoffroy opère un transfert de la résidence ducale, abandonnant le site de Saint-Projet pour lui préférer un site suburbain, destiné à contrôler le trafic fluvial à la confluence de la Garonne et du Peugue.

Le site de Saint-Projet retourne dans la pénombre documentaire jusque dans la seconde moitié du XII ${ }^{\mathrm{e}}$., alors que la tour de l'Arbalesteyre est un peu plus documentée, au gré des arrêts du duc ou des demandes de réparation de son gardien. C'est en 1187 que Saint-Projet revient sur le devant de la scène, dans une notice du cartulaire de Sainte-Croix de Bordeaux, abbaye bénédictine du sud de la ville, à travers un énigmatique "mai" (madum). Le texte rapporte les clauses d'un accord survenu le 11 juin de cette année entre l'abbé de Sainte-Croix et son voisin, le seigneur de Centujan, sur les modalités de règlement des futurs conflits entre leurs deux seigneuries :

"En outre, sur tout ce qui concerne le ruisseau et les moulins [qu'ils se disputent], l'abbaye n'aura pas à faire le mai (facere madum) à Baudouin de Centujan ou à personne le représentant. Il est reconnu par les deux parties que les moines n'auront qu'à envoyer un ou deux d'entre eux, le jour du mai (die madum), à Saint-Projet, y prêter serment de foi à Baudouin et Amanieu au nom des alleux [i.e. en cas de plainte sur les alleux] et, remettant caution, s'en retourner à l'abbaye. Puis, si lui-même ou un autre devait déposer une plainte à propos des fonds allodiaux, l'abbaye ne pourrait être assignée nulle part ailleurs qu'à Sainte-Croix. Ici la cause serait traitée selon le droit de la mer dans les mains de Baudouin. [L'abbaye] n'est tenue de répondre devant Baudouin en raison du mai que sur les fonds allodiaux" ${ }^{26}$.

23. Archives historiques du département de la Gironde (désormais AHG), t. 3, n II, t. 49, n IX et Archives départementales de la Gironde (désormais AD Gironde), H 2013.

24- AD Gironde, G. 334.

25- Pour les références, voir Boutoulle 2003, 64.

26- Ducaunnès-Duval, éd. 1892, n LXII : Preterea de omnibus his que pertinent ad aquam, sive ad molendinos hujus aque, dicta ecclesia non debet facere madum ipsi Baudoino, vel in aliquo nomine ejus aque respondere. Recognitumque fuit a partibus et placuit ipsi ecclesie et Baudouino et Amanevo quod, die madi, unus vel duo de monachis Sancte Crucis apud Sanctum Perjectum debet accedere, et fidantiam nomine allaudiorum tantum illi dare, statim recedere; et si ipse vel alius de fundo allaudiorum questionem moverit ea die debet dies [...] apud Sanctam Crucem eidem ecclesie assignari, et ibidem et non alibi in manu Baudoini jure mari causa tractari et finiri et de nullo nisi de fundo allaudiorum ecclesia eidem Baudoino ratione madi tenetur respondere. Le madum dont il est question ne nous semble pas être les pousses printanières que l'on ramasse au mois de mai, parfois dans un but festif. La localisation à Saint-Projet, de l'ancien palais ducal, et la vocation judiciaire de ce madum, nous met plutôt face à une des sessions de l'ancien tribunal comtal, le mallus publicus, dont nous n'avons par ailleurs aucune autre trace. 
De cette clause complexe, il apparaît que Baudouin a la connaissance des conflits portant sur les alleux de sa seigneurie, mais que ceux-ci sont traités à Saint-Projet. L'abbaye obtient que les causes la concernant soient jugés à SainteCroix, devant Baudouin, par la procédure inconnue du "droit de la mer". Quant au "mai de Saint-Projet", il s'agit probablement d'une session du tribunal public (mallus pour madum), puisqu'on y traite des conflits sur les alleux. Il porte le nom des assemblées du haut Moyen Âge, les "champs de mai" au caractère militaire et politique bien connu et dont on n'a, pour le Bordelais, aucune preuve de l'existence avant $1187^{27}$. Le maintien d'une fonction judiciaire à Saint-Projet peut expliquer l'absence de l'aula ou de la Sala dans les donations de 1070-1072 : à l'époque, Guy Geoffroy ne l'a probablement pas aliénée.

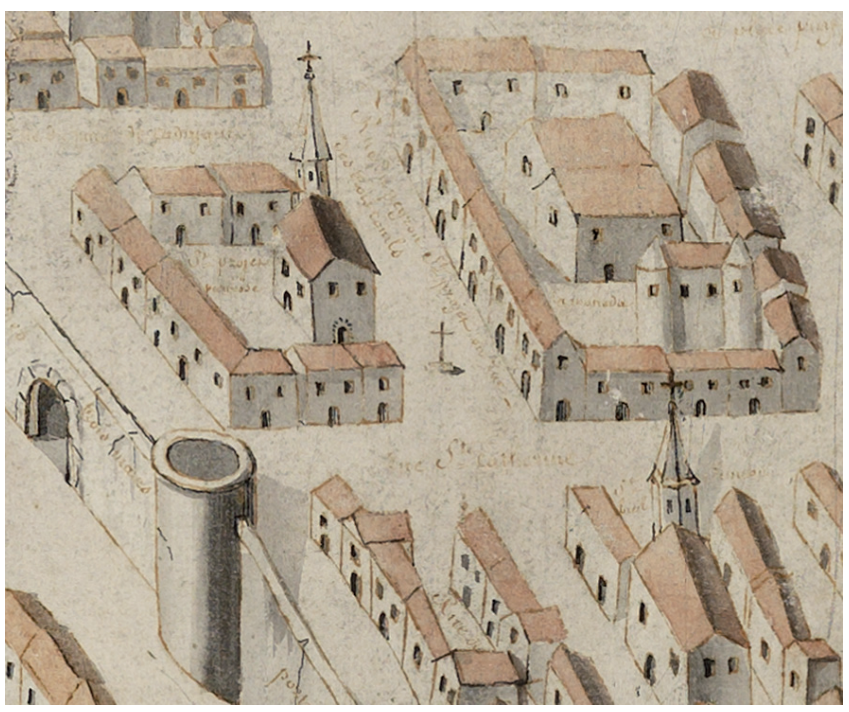

Fig. 6. Saint-Projet paroisse et La moneda (AM Bordeaux, 40B8 30PP9, cl. B. Rakotomanga).

Un nouveau jalon textuel confirme la permanence de cette fonction publique et judiciaire dans un XIII $\mathrm{s}$. bien avancé. Le 22 juillet 1228, le roi d'Angleterre et duc d'Aquitaine Henri III confie à Arnaud de La Lande, citoyen de Bordeaux, prévôt de la monnaie, la domus monete. Le même mandement précise que l'atelier monétaire est à proximité de l'aula royale ${ }^{28}$. À notre connaissance, c'est la première mention de la proximité géographique de l'aula avec l'atelier monétaire, dont les textes de la même époque $(1233,1262)$ nous disent qu'il est effectivement à Saint-Projet ${ }^{29}$.

La meilleure compréhension de l'accord de 1187 nous conduit à reconsidérer la continuité de la vocation publique de Saint-Projet. En 2003, nous avions envisagé que le transfert de 1070 était total et qu'il y avait eu, en 1187 ou peu avant, et jusqu'en 1254, une sorte de "retour aux sources", en raison des nuisances que devaient occasionner, soit le délabrement du site de l'Ombrière (témoignages de son mauvais état en 1147) soit le début d'un plus vaste chantier ${ }^{30}$. Aujourd'hui, s'impose la possibilité d'une permanence de l'aula de Saint-Projet, au-delà de 1070, avec sa vocation publique et judiciaire. L'hypothèse oblige à penser la disjonction des fonctions judiciaires et résidentielles sur deux sites différents.

Tentons maintenant d'utiliser les informations disponibles pour localiser les éléments de ce palais. La seule représentation de l'atelier monétaire figure sur une copie d'une vue chorographique réalisée au XIXe s. à partir d'un portrait perdu de la ville fait dans les années $1525-1535^{31}$ (fig. 6). La moneda y est localisée à l'arrière d'un

27- Wallace-Hadrill, éd. 2001, c. 42, 186, lin. 28 (42), mentions du "champs de mai" durant l'époque carolingienne : (...) omnes obtimates Francorum (...) ad campo Madio pro salutem patrie et utilitatem Francorum tractandum, placito instituto (...). Ibid., c. 47, 189, lin. 19 (47) : (...) commoto omni exercito Francorum (...) cum Francis et proceribus suis placitum suum campo Madio tenens. Ibid., c. 49, 190, lin. 28 : Iterum campo Madio, sicut mos erat, ibidem tenere iubet.

28- Maxwell Lyte, éd. 1902, 67 : Dominus rex commisit Ernaldo de Landa civi Burdegale et preposito monete Burdegale, qui bene et fideliter servivit domino J. regi et domino regi, domum domini regis de monetaria junctam aule domini regis in Burdegala; Lee, éd. $1903,196$.

29. Ducaunnès-Duval \& Barckhausen, éd. 1867, 368-370 ; Barckhausen, éd. 1890, 336-338 : item dicimus quod omne plate Sancti Prejecti prout extenduntur ab ecclesia sancti Prejecti, usque ad domum in qua moneta fieri consuevit et via publica est utroque latere sunt paduentum (...) e vi quans los maysons de Sent Progeit, qui esta costa la plassa, fade hom la moneda (...) et bit que a la mayson de la moneda de Sent-Proget fade hom la moneda (...) et que la mayson Sent Proget es aux obreys de la moneda es. AD Gironde, 4 J 73, (cartulaire de Saint-André), fol. 51, 1233) domum in parrochia Sancti Perjecti, juxta domum monete in magna rua de Porta Medulca. Francisque, éd. 1885, n²109, 2139 et 2651 ; Bémont, éd. $1896, n^{\circ} 4215$ et 4217 .

30- Le 26 octobre 1255, le roi ordonne au connétable de Bordeaux de faire construire l'aula Burdegale avec les revenus prélevés sur la grande coutume des vins. Bémont, éd. 1896, n 4674 : Mandatum est magistro B. constabulario Burdegale quod aulam Burdegale de peccunia costume majoris operari faciat.

31- Jean-Courret 2006b. 
alignement de maisons donnant sur la rue Sainte-Catherine ce qui recoupe les informations que l'on a des textes du XIII ${ }^{\mathrm{e}}$ s. Sur cette vue, la moneda est représentée comme un haut édifice encadré de deux tours d'angle en façade. Si l'on suit la confirmation de la donation du tiers des revenus de l'atelier monétaire évoqué plus haut, qui établit l'équivalence camera-monete, c'est probablement là qu'il faut localiser l'ensemble résidentiel de ce palais (fig. 7). Le capitulaire De Villis nous enseigne que la camera sert également à entasser le trésor ${ }^{32}$.

La capella palatii de 1072-1077 est certainement l'église Saint-Projet donnée à Saint-Martin du MontJudaïque, dédiée à un évêque auvergnat de la fin du VII ${ }^{\mathrm{e}} \mathrm{s}^{33}$. Mais en 1179 , d'après une bulle du pape Alexandre III, elle fait partie des ecclesiae relevant de la cathédrale situées dans la moitié sud de la cité ${ }^{34}$. L'église du XVIII ${ }^{e}$ s. sous laquelle on a reconnu des vestiges gallo-romains, est placée au sud de la place SaintProjet. Pour localiser la Salle, nous disposons du nom ancien de l'actuelle impasse Saint-Projet, autrefois appelée rueta de La Sala ou rueta de Noaillan ${ }^{35}$. Un acte foncier de 1369 évoque justement la sala de Noalhan, confrontant une maison située rue Sainte-Catherine ${ }^{36}$. Sur le plan, l'espace au nord de la place Saint-Projet entre l'impasse et la rue Sainte-Catherine est large d'une dizaine de mètres, ce qui correspond aux dimensions des façades d'autres palais contemporains (Paderborn 10 m, Tilleda 9,50 m, Douai-la-Fontaine $13 \mathrm{~m})^{37}$. La façade de cette aula donnait probablement sur la place Saint-Projet. L'atrium mentionné par la donation de 1070 est soit une salle d'entrée, soit un portique. La description du fisc d'Annapes (c. 800) confirme l'association d'un tel équipement avec une salle royale et des camerae ${ }^{38}$. Pour l'instant nous ne sommes pas parvenus à localiser son emplacement. Enfin, le puits donné en 1070 correspond certainement à l'un des trois puits identifiés dans les limites de la paroisse Saint-Projet : le putz de Santa Gemma dans la rue du même nom (attesté en 1307, au carrefour des rues Guérin et du Loup), un puits que signale Léo Drouyn rue de la Moneda, et le Putz de Saint-Projet, sur la place éponyme (attesté en 1375) ${ }^{39}$.

D'ores et déjà, c'est un schéma de dispersion qui domine et non pas de concentration fonctionnelle des locaux sous un même toit (comme à Douai-la-Fontaine). À Saint-Projet (fig. 7), le pôle noble de la résidence semble éclaté en plusieurs bâtiments, situés à faible distance les uns des autres, comme à Fécamp où l'église est aussi rejetée. Finalement, d'après les textes, nous aurions un groupement important au nord de la place Saint-Projet, avec la Salle, la camera et l'atelier monétaire, formant un ensemble majeur s'étendant sur environ $125 \mathrm{~m}$ (de la place Saint-Projet à l'impasse de la Monnaie au bord de la Devèze) et sur $30 \mathrm{~m}$ de large. C'est précisément dans ce premier ensemble que sont localisés les padouens de Saint-Projet en 1262 : l'enquête dit que "tout est padouen, depuis la place et l'église Saint-Projet jusqu'à la domus monete, ainsi que la voie publique avec ses deux côtés" 40 . L'église est positionnée au sud de la place, elle n'a donné lieu ni à une collégiale ni à une abbatiale, alors que les églises de palais prennent souvent une fonction de collégiale. La position de ce lieu de culte près d'une porte évoque plutôt une chapelle de porte. Les portes donnant sur la place de Saint-Projet, seuls éléments de l'enceinte signalés par les textes, ressemblent à celle que mentionne la descriptio du fisc d'Annapes, unique partie en pierre de l'enceinte d'une curtis ${ }^{41}$.

\footnotetext{
32. Renoux 1987, 237-271.

33. Évêque de Clermont mort en 676, saint Priest est connu sous le nom de saint Prix en Île-de-France, saint Preils en Saintonge et saint Projet en Bordelais.

34- $A H G$, t. $13,358-360$.

35- Drouyn 1874.

36- AD Gironde, G. 340, fol. 40 (1369) ; Rayou 1994, 1.

37. Renoux 1987, 289 et sq; Renoux 2002 et Zotz 2002.

38. La Roncière et al. 1969, 227-228.

39. Jean-Courret 2006c.

40- Ducaunnès-Duval \& Barckhausen, éd. 1867, 368-370 ; Barckhausen, éd. 1890, 336-338. Les autres églises et places intra-muros de l'enquête sont la cathédrale Saint-André (platea Sancti Andree) et l'église Saint-Pierre (ecclesia Sancti Petri). On appelle padouens les terres publiques ouvertes à l'usage des communautés d'habitants; par extension, le padouen est aussi l'usage de ces espaces.

41- La Roncière et al. 1969 : "la cour fortement défendue par une haie, avec un portail de pierre, et par-dessus un solier servant de dépense" (curtem tunimo strenue munitam, cum porta lapidea, et desuper solarium ad dispensandum).
} 


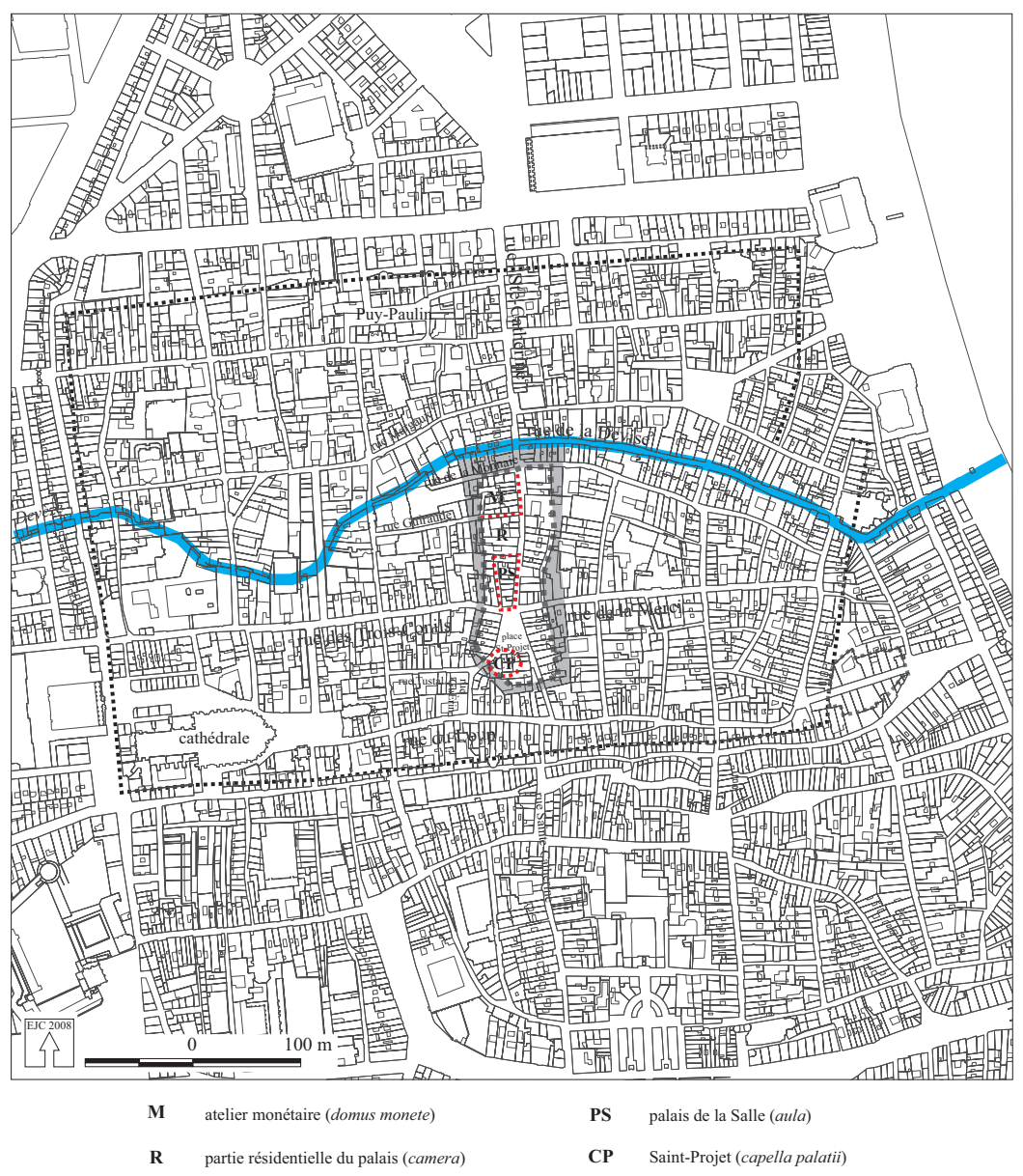

Fig. 7. Localisation des sites du complexe palatial de Saint-Projet (cartographie É. JeanCourret).

\section{PERSPECTIVES}

Bien des questions restent en suspens. Il conviendrait d'abord d'affiner la connaissance de la structure interne de cet ensemble palatial. Ces éléments semblent avoir été disposés en enfilade, à moins qu'avec la cour dont il est question nous ayons une disposition différente (en $\mathrm{L}$ ou en équerre plus qu'un système à cour centrale). Nous n'avons pas trouvé dans la documentation ultérieure d'allusion aux possessions de Saint-Sever à Saint-Projet. Qu'est-ce que l'abbaye gasconne a fait de ses droits ${ }^{42}$ ? Par ailleurs, le label palatial pose question, car il est rarissime dans les principautés de cette période ${ }^{43}$. Est-il à mettre sur le compte de la personnalité hors du commun de l'abbé Grégoire de Montaner, abbé de Saint-Sever et de Saint-Genis de Lectoure, évêque de Lescar puis de Dax (1028-1072), comme si pour renforcer la puissance de son abbaye, il avait fait changer la qualification d'une curtis ducale, ou est-ce lié aux fonctions intrinsèques des bâtiments qu'il a reçus? On se demande aussi ce qui explique le maintien d'une aula à Saint-Projet après 1070. Il n'est pas exclu que cela soit

42- Le principat de Richard Cœur de Lion semble avoir été le théâtre d'une redistribution des cartes. AD Gironde, G 75 fol. 7 : le prieur du Mont-Judaïque dessert la capella castri Burdegale depuis que Richard, comte de Poitiers, la lui affecta par un chirographe "afin d'augmenter les revenus dudit couvent qui avait eu à souffrir de l'expulsion des juifs d'Aquitaine".

43. Renoux 1992 ; Renoux 2002 et Zotz 2002. 
la conséquence de l'attachement de la population de la ville et de ses environs à ce vieux tribunal public et des réticences bien connues des hommes libres à voir leurs lieux de justice coutumiers être transférés. La question nous amène à réexaminer les raisons de la réussite du second transfert, puisqu'en 1254 le roi-duc peut faire construire une nouvelle aula regis à l'Ombrière et à nous interroger sur le rôle de la municipalité en cette occasion, puisque c'est à ce moment que l'ensemble palatial est converti en padouens ${ }^{44}$.

La question des origines de cet ensemble est évidemment un problème lancinant. L'enceinte de la curtis dans laquelle il s'inscrit avait-elle vocation à protéger les bâtiments publics contre des attaques venues du fleuve? On ne peut, dans cette hypothèse, s'empêcher de penser aux incursions scandinaves dont Bordeaux a souffert en 848, 851 et 863-864. La ville qui a été prise par les Normands et qui leur a semble-t-il servi un temps de base, aurait été, aux dires de l'archevêque Frotaire, désertée d'une partie de ses habitants ${ }^{45}$. Ne pouvant mesurer l'impact de ces incursions à Bordeaux, il faut observer ailleurs ce que l'arrivée des Scandinaves a impulsé. Le cas de Rouen, éclairé par les fouilles de J. le Maho, fournit un parallèle très intéressant. Là, dans le quartier de la cathédrale, on note une densification du bâti à la fin du IX ${ }^{e}$ s., probablement causée par un afflux de réfugiés fuyant les Scandinaves. Une telle refondation, selon l'archéologue, ne peut avoir été conduite que sous l'égide du pouvoir royal ${ }^{46}$.

Dans cette quête des origines, on ne peut s'empêcher d'apporter, sans trop savoir quoi faire de ce type d'information, le témoignage d'un dernier texte, un extrait de la Chronique dite Saintongeaise. Ce récit a été rédigé dans les premières années du XIII $\mathrm{e}$ s., probablement, selon son éditeur, par un clerc de Saint-Seurin de Bordeaux. Il s'agit d'une œuvre littéraire, qui reprend la traduction du Pseudo-Turpin faite par Nicolas de SaintLis (v. 1202-1205). L'auteur, qui s'exprime dans un saintongeais émaillé de gasconismes, introduit dans son texte de fréquentes interpolations, puisées dans les archives de Saint-Seurin ou dans des traditions locales épiques inconnues, sur les exploits d'un roi Charles contre les Sarrasins en Saintonge, en Agenais et en Bordelais. C'est un texte en prose qui est à la fois un discours hagiographique et un texte épique. Le passage qui nous intéresse décrit la manœuvre conduite par Roland, le palatin de Charlemagne, accompagné de Turpin, l'archevêque de Reims, contre les "Sarrasins" qui tiennent Bordeaux.

"Ensuite Roland et Turpin s'en allèrent vers la ville (...). Roland et ses compagnons attaquèrent très durement ; un sarrasin dressa une échelle contre la salle où les dames s'étaient retirées, et en commença l'ascension. Mais Roland lança un pieu et tua le sarrasin qui tomba, alors que le pieu se ficha dans le mur. Quand les Sarrasins virent cela, ils s'en furent et Roland trancha les gonds de la porte. Il avança jusqu'à une eau qui s'appelle la Devèze. Il trouva Guolias que Aiguolanz [le roi de Bougie] y avait laissé vingt mille Sarrasins. Ici la bataille fut grande, et ils tuèrent dix mille Sarrasins" $\$$.

Il est toujours délicat de faire le tri dans un récit comme celui-ci, entre la part de fiction et les faits qui se sont réellement passés, car, en matière de mémoire, les communautés de clercs, celle de Saint-Seurin comme les autres, savent mixer plusieurs niveaux d'informations, à l'exemple du cartulaire de Saint-Seurin qui mêle actes authentiques ou réécrits avec des constructions à finalités politiques. Jusqu'à aujourd'hui, aucun historien n'a prêté attention à cette mention de bataille en plein cœur de la ville, près de la Devèze, entre des Carolingiens et des adversaires présentés comme des Sarrasins ${ }^{48}$. Avec ce que l'on sait maintenant de cet espace central de la cité, il ne faut pas exclure que ce passage de la Chronique Saintongeaise soit le porteur d'une mémoire particulière, "l'écho abâtardi de quelque tradition locale" (J.-C. Cassard). Pour les chantiers de fouille à venir, ce n'est pas la piste la moins stimulante...

44- Lavaud \& Jean-Courret 2009, t. 2, 86-87.

45- Recension des sources dans Boutoulle 2008.

46- Le Maho 2005 et 2006.

47. De Mandach, éd. 1970, 287-288.

48- La Chanson d'Aiquin, seule chanson de geste composée en Bretagne au XIII s., confond les Sarrasins et les Vikings (Cassard 1996, 104). 


\section{Sources et bibliographie}

Archives historiques du département de la Gironde, édité par la Société des Archives historiques de la Gironde t. III (1861), t. XIII (1872), t. XLIX (1914).

Barckhausen, H., éd. (1890) : Livre des Coutumes, Archives municipales de Bordeaux, t. V, Bordeaux.

Bauduin, P., dir. (2005) : Les fondations scandinaves en Occident et les débuts du duché de Normandie : colloque de Cerisy-la-Salle, 25-29 septembre 2002, Caen.

Bémont, C., éd. (1896) : Rôles gascons, suppl. au t. I, 1254-1255, Paris.

De Mandach, A., éd. (1970) : Chronique dite Saintongeaise : texte franco-occitan inédit "Lee" : à la découverte d'une chronique gasconne du xIIF siècle et de sa poitevinisation, Genève-Paris.

Ducaunnès-Duval, A., éd. (1892) : "Cartulaire de l'abbaye SainteCroix de Bordeaux", Archives historiques du département de la Gironde, XXVII.

Ducaunnès-Duval, A. et $\mathrm{H}$. Barckhausen, éd. (1867) : Livre des Bouillons, Archives municipales de Bordeaux, t. I, Bordeaux.

Francisque, M., éd. (1885) : Rôles gascons, I, 1242-1254, Paris.

Lee, H. B. (1903) : Patent Rolls of the Reign of Henry III, Preserved in the Public Record Office, II, 1225-1232, Londres.

Maxwell Lyte, H. C., éd. (1902) : Close Rolls of the Reign of Henry III, Preserved in the Public Record Office, I, 1227-1231, Londres.

Pon G. (2006) : "Diplôme de Gui-Geoffroy-Guillaume concernant un palais à Bordeaux", Revue Historique du Centre-Ouest, V, 421.

Pon, G. et J. Cabanot, éd. (2010) : Chartes et documents hagiographiques de l'abbaye de Saint-Sever (Landes), 9881359, Dax.

Wallace-Hadrill, J., éd., O. Devillers et J. Meyers, trad. (2001) : Fredegarius schol., Chronicarum continuationes, MGH, SS rer. Merov., t. II, Turnhout.

Barraud, D, F. Hautefeuille et C. Rémy, dir. (2005) : Résidences aristocratiques, résidences du pouvoir entre Loire et Pyrénées, $x^{e}-X V^{e}$ siècles: recherches archéologiques récentes, 1987-2002: Actes du colloque de Pau 3- 5 octobre 2002, Archéologie du Midi médiéval Suppl. 4, Carcassonne.

Bourgeois, L., dir. (2008) : Fortification et résidences des élites du haut Moyen Âge entre Loire et Garonne, PCR, rapport.

Bourgeois, L. et C. Rémy, dir. (2014) : Demeurer, défendre et paraître: orientations récentes de l'archéologie des fortifications et des résidences aristocratiques médiévales entre Loire et Pyrénées, Actes du colloque de Chauvigny, 14-16 juin 2012, Chauvigny.

Boutoulle, F. (2003) : "Enceintes, tours, palais et castrum à Bordeaux, du xie siècle au début du xille siècle, d'après les textes", Revue archéologique de Bordeaux, XCIV, 59-77.

- (2008) : "'Par peur des Normands'. Les Vikings à Bordeaux et la mémoire de leurs incursions. État des sources", Revue archéologique de Bordeaux, IC, 28-38.

Cassard, J.-C. (1996) : Le siècle des Vikings en Bretagne, Paris.

Drouyn, L. (1874) : Bordeaux vers 1450, Bordeaux.

Gaidon-Bunuel, M.-A. (2002) : Observations archéologiques aux $n^{\circ} 19$ et 25 de la rue des Trois-Conils, DRAC Aquitaine SRA, rapport coté $\mathrm{BX}$ 06/02.
Gauthiez, B. (2003) : Espace urbain, vocabulaire et morphologie, coll. Inventaire général des monuments et des richesses artistiques de la France, Paris.

Higounet, C., dir. (1963) : Histoire de Bordeaux, II, Bordeaux pendant le haut Moyen Âge, Bordeaux.

Jean-Courret, É. (2006a) : La morphogenèse de Bordeaux des origines à la fin du Moyen Âge : fabrique, paysages et représentations de I'Urbs, thèse de doctorat, université de Bordeaux III.

- (2006b) : "Civitas Burdegalensis geniuna descriptio : une représentation de Bordeaux vers 1525-1535", Revue archéologique de Bordeaux, XCVII, 57-87.

— (2006c) : "De l'estey au putz. Pollutions et équipements d'eau à Bordeaux (fin XIII'-xve siècle)", in : L'eau en Bordelais de l'Antiquité à nos jours, Revue Historique de Bordeaux et du département de la Gironde, 9-10, 47-76.

— (2010): "Aquae versus Acqs : seize siècles de la fabrique urbaine de Dax", Aquitania, 26, 159-206.

- (2014) : "Les pouvoirs de l'enceinte. Historiographie nationale et nouvelles propositions autour du cas de Bordeaux (ivexII siècles)", in : Bourgeois \& Rémy, dir. 2014, 391-411.

Jullian, C. (1887-1890) : Inscriptions romaines de Bordeaux, t. I, Bordeaux.

Lavaud, S. et É. Jean-Courret, dir. (2009) : Atlas historique de Bordeaux, Atlas historique des villes de France, Bordeaux.

Le Maho, J. (2005) : "Les Normands de la Seine à la fin du ix siècle", in : Bauduin, dir. 2005, 161-179.

-(2006): " Le groupe épiscopal de Rouen, des temps paléochrétiens à l'époque des raids vikings $\left(\mathbb{V}^{\mathrm{e}}-\mathrm{I} \mathrm{X}^{\mathrm{e}} \mathrm{s}\right.$.). Le témoignage des textes et de l'archéologie ", in : 396-1996-XVle centenaire de la cathédrale Notre-Dame de Rouen, colloque international, 5,6, et 7 décembre 1996, Rouen, 201-224

La Roncière, C.-M., R. Delort et M. Rouche (1969) : L'Europe au Moyen Âge, t. 1, Paris.

Maillé, A. (1960) : Recherches sur les origines chrétiennes de Bordeaux, Paris.

Marin, A. (2001a) : Église Saint-Projet, 97 rue Sainte-Catherine, Bordeaux (Gironde), Bureau d'études Hades-DRAC Aquitaine SRA, rapport coté BX 11/07.

— (2001b) : "Bordeaux. 97 rue Sainte-Catherine. Église SaintProjet", Bilan scientifique du Service régionale d'Archéologique d'Aquitaine, 62-63.

Nony, D. et H. Redeuilh (1955-1956) : “ Découvertes archéologiques places Saint-Projet, Saint-Pierre et du Parlement à Bordeaux en 1955-1956 ", Bulletin et mémoires de la Société archéologique de Bordeaux, LIX, 118-121.

Oexle, O. G. et J.-L. Schmitt, dir. (2002) : Les tendances actuelles de I'histoire du Moyen Âge en France et en Allemagne, Actes de colloques de Sèvres (1997) et Gottingen (1998), Paris.

Parisse, M. et X. Barral i Altet, dir. (1992) : Le roi de France et son royaume autour de I'an Mil : actes du colloque Huques Capet 987-1987. La France de I'an Mil, Paris-Senlis, 22-25 juin 1987, Paris. 
Rayou, C. (1994) : La paroisse Saint-Projet de Bordeaux, de 1300 à 1553, TER, Université de Bordeaux-Montaigne.

Renoux, A. (1987) : Fécamp, du palais ducal au palais de Dieu : bilan historique et archéologique des recherches menées sur le site du château des ducs de Normandie : "f siècle a.C.-xv\|l siècle p.C., Paris.

— (1992) : "Palais capétiens et normands à la fin du xe et au début du xie siècle", in : Parisse \& Barral i Altet, dir. 1992, 179-191.
— (2002) : "Palais, cours et résidences", in : Oexle \& Schmitt, dir. 2002, 351-356

Sansas, P. (1861) : "Notes sur quelques sépultures récemment découvertes à Bordeaux", in : Congrès scientifique de France, $28^{\mathrm{e}}$ session, Bordeaux, septembre 1861, t. IV, 469.

Zotz, T. (2002) : "L'étude des palais royaux en Allemagne", in : Oexle \& Schmitt, dir. 2002, 307-326. 